\title{
Simultaneous Tuning of Power System Stabilizers Installed in DFIG-Based Wind Generation
}

\author{
Ângelo Mendonça, and J. A. Peças Lopes, Senior Member, IEEE
}

\begin{abstract}
This paper addresses the problem of large wind power integration and its impact on damping of the electromechanical modes of oscillation. The viability of the doubly fed induction generator (DFIG) supplying additional damping is discussed and power system stabilizers (PSS) installed in DFIG wind power generators are simultaneously tuned to increase damping levels of a multimachine system.
\end{abstract}

Index Terms - Doubly fed induction generator (DFIG), power system stabilizer (PSS), small signal stability.

\section{INTRODUCTION}

$\mathrm{L}$ ARGE scale wind power integration has a significant im$u_{\text {pact }}$ on the operation of power systems, including on the levels of oscillations damping [1]. Hence, when connecting wind power to a system there is the need to guarantee that transient performance is not reduced and problems of small signal stability do not arise.

The most frequent cause of this kind of instability is the lack of damping of the so called electromechanical modes of oscillation, which are related with low frequency $(0.1-2 \mathrm{~Hz})$ power oscillations that occur in the rotor of synchronous machines, essentially due to controllers settings and the efforts to transmit bulk power through weak transmission lines [2], [3]. It has been demonstrated that wind generation has some impacts on the damping levels of a power system, and the nature of the impacts depends on factors like wind generation conversion technology or network configuration [1].

Power system stabilizers (PSS) installed in the excitation of synchronous generators (SG) is still one of the most cost effective solutions capable of providing supplementary damping. However, in a system with large share of wind power generation these devices may no longer be able of providing the necessary additional damping. For example, if the synchronous generators which have these devices installed are switched off to accommodate the wind power, their damping contribution is obviously lost. It is therefore desirable that wind power, and in particular doubly fed induction generators (DFIG), may in the

This work was supported by the Fundação para a Ciência e a Tecnologia (FCT) of Portugal Grant SFRH/BD/18469/2004.

$\hat{A}$. Mendonça is with the Power Systems unit of INESC Porto, Porto, Portugal (e-mail: angelo.mendonca@inescporto.pt).

J. A. Peças Lopes is with the Power Systems unit of INESC Porto and with the Department of Electrical and Computer Engineering, Porto University, Porto, Portugal (e-mail: jpl@fe.up.pt). future be able to supply supplying damping as an ancillary service, as proposed in [4],[5].

The DFIG can be used to improve the overall damping of the electromechanical modes of oscillation, as many FACTS controllers do, through modulation of its power output. But like in the case of FACTS that is not straightforward, because electronic devices are not directly involved in the electromechanical oscillations [3].

The effectiveness of a PSS, and consequently the contribution of the machine to the damping of the modes of oscillation, depends on appropriate tuning. It is therefore essential to find a solution capable of providing satisfactory behavior. The approach adopted in this research for tuning the PSS consists of solving an optimization problem that pursues the minimization of the total PSS control actions, provided that a minimum acceptable damping is achieved.

It is the objective of this research to analyze the contribution of DFIG units to the problem of small signal stability and to take advantage of control strategies that employ the flexibility provided by these machines to increase the damping of electromechanical oscillations. The study was conducted using mainly modal analysis, which is a powerful tool that has been used for a long time to characterize oscillations and design controllers. The results were validated afterwards, performing nonlinear time domain simulations. Modal analysis was carried out using a MATLAB ${ }^{\mathrm{TM}}$ program developed for that purpose. Nonlinear time domain simulations were carried out using $\mathrm{PSS}^{\mathrm{TM}} \mathrm{E}$ software package.

The following sections describe the mathematical model of the DFIG, the configuration of the PSS, and the algorithm adopted for PSS tuning. Results of a study case are then presented and finally conclusions are outlined.

\section{MATHEMATICAL MODELS}

The dynamic and steady state equations that describe the behavior of the DIFG-based wind generator are well described in the literature. In this research, the mathematical model proposed in [6] was used. This detailed model represents the fundamental dynamics of a single cage induction machine, wind turbine with pitch angle control and PI controllers of the rotor side static converter, as shown by the control scheme of Fig. 1 . Detailed description of generator and wind turbine parameters can also be found in [6]. Note that an auxiliary stabilizing signal $V_{p s s}$, output of the PSS, is summed to the rotor quadrature voltage in the active power control loop. This signal creates a 


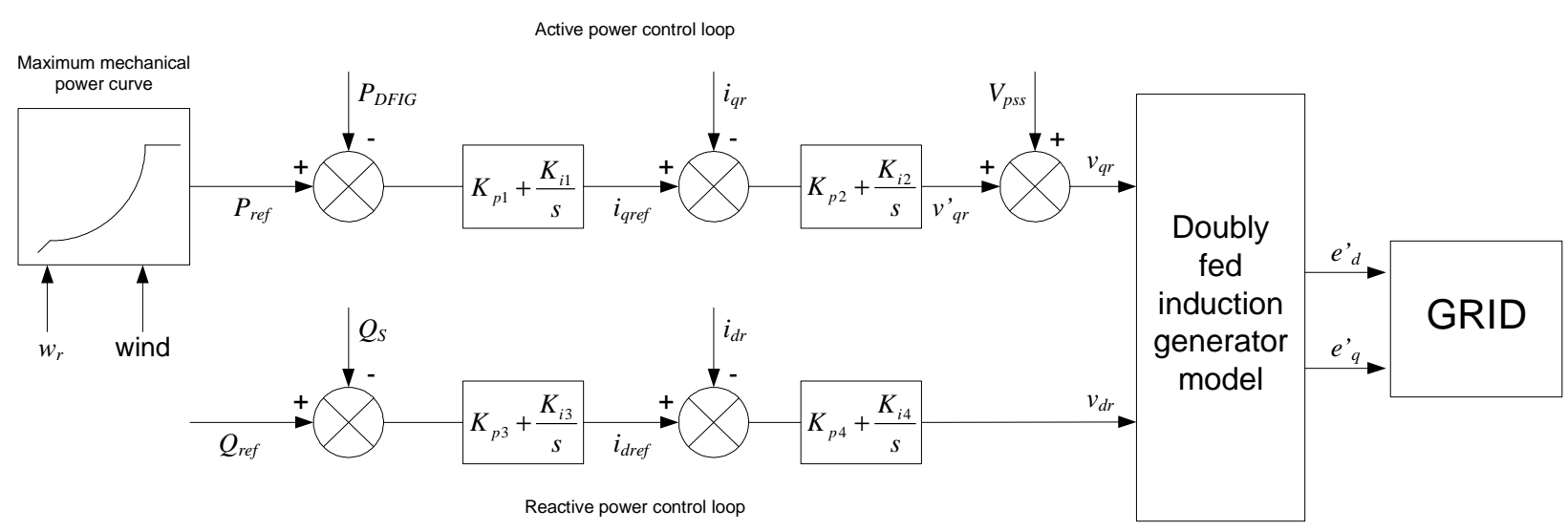

Fig. 1. Control scheme of the rotor side PI controllers with PSS.

component of generator damping torque that is in phase with rotor speed by modulation of the active power [5]. In order to modify the mode of oscillation, the chosen input and output signals should provide adequate observability and controllability. The DFIG acts to amplify the power of the controller, as synchronous generators and FACTS devices [3].

A linearized model in a state space form, suitable for modal analysis, was derived from the detailed model. Assuming that the pitch angle is constant during the studied period, only the equations of the generator and PI controllers need to be linearized. The complete model contains seven state variables. Three correspond to the induction machine: speed deviation, and direct and quadrature components of the generator internal voltage. Four state variables correspond to the PI controllers.

The damping conditions associated to each mode of oscillation are determined calculating the eigenvalues of the state matrix exploiting QR method routines from MATLAB ${ }^{\mathrm{TM}}$.

The PSS configuration used in this research is shown in Fig. 2, where $K$ is the PSS gain, $T_{w}$ is the washout time constant (to prevent steady changes in speed from modifying the field voltage), and $T_{1}$ to $T_{4}$ are the time constants of the lead-lag blocks (to provide the necessary phase compensation).

This type of stabilizer is also adopted in this research to be installed in synchronous generators. In this case the PSS output is summed to the reference of the excitation system.

\section{PSS TUNING}

PSS parameters are determined solving an optimization problem that models the operational requirements. The adopted formulation is derived from the method presented in [7] that pursues the minimization of the PSS gain, provided that minimum acceptable damping (in this research set to 15\%) is achieved. This approach has the benefit of producing low stabilizer gains that reduce the effect of limiting in the stabilizer and other controllers [8]. Although developed for tuning PSS installed in synchronous generators, the use of this approach for tuning PSS to install in DFIG is straightforward. It should be noted however that care must be taken so that the installation of the PSS does not decrease the performance of the machine by moving the control modes of the DFIG.

Mathematically, the problem can be described by the fol-

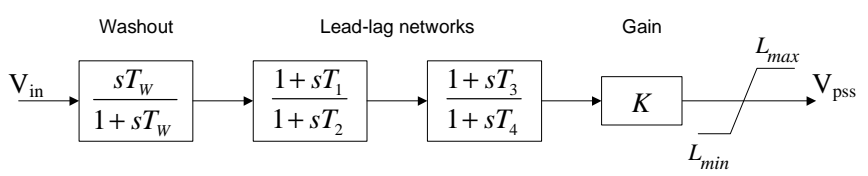

Fig. 2. Classical power system stabilizer.

lowing minimization of the quality index $Q$.

$$
\begin{aligned}
& \min \quad Q(\underline{X})=\sum_{i=1}^{n m} \sum_{j=1}^{n g} w_{j i} K_{j} \\
& \text { subject to } \quad \boldsymbol{S}_{i} \geq \boldsymbol{\zeta}_{i \text {.min }} \\
& \left|\Delta f_{i}\right| \leq \Delta f_{i . \min } \\
& \underline{X}_{j \text { max }} \geq \underline{X}_{j} \geq \underline{X}_{j \text {.min }}
\end{aligned}
$$

Where:

$\underline{X}$ is the solution of the problem (set of control variables to be used in each PSS to be installed);

$w_{j i}$ are the weighting factors;

$K_{j} \quad$ is the PSS gain of the $j$-th generator;

$n g$ is the total number of generators;

$\mathrm{nm}$ is the number of modes;

$\zeta_{i} \quad$ is the damping of the $i$-th eigenvalue;

$\Delta f_{i}$ is the frequency change of the $i$-th eigenvalue.

The objective function described by (1) consists of the sum of the PSS gains, subject to a set of restrictions: a) damping of the modes of oscillation should be higher then a given bound, described by (2); b) frequency should be within a given bound, described by (3); and c) control variables should be within allowable physical limits, described by (4).

The transfer function of the PSS, as a function of the control variables (solution of the problem), is shown in (5).

$$
V_{p s s}(s)=K \frac{s T_{W}}{1+s T_{W}} \cdot\left(\frac{1+s T_{n}}{1+s T_{n} / \alpha}\right)^{N} \cdot V_{i n}(s)
$$

Where $K$ is the PSS gain and $T_{w}$ the washout time constant. The control variables are, for each PSS, the gain $K$, the number of lead-lag blocks $N$ and the time constant values $\left(T_{n}=T_{l}\right.$ and $\alpha=T_{1} / T_{2}$ ) of each of these blocks. Limits are also imposed to the gains of the stabilizers and to the time constants in 


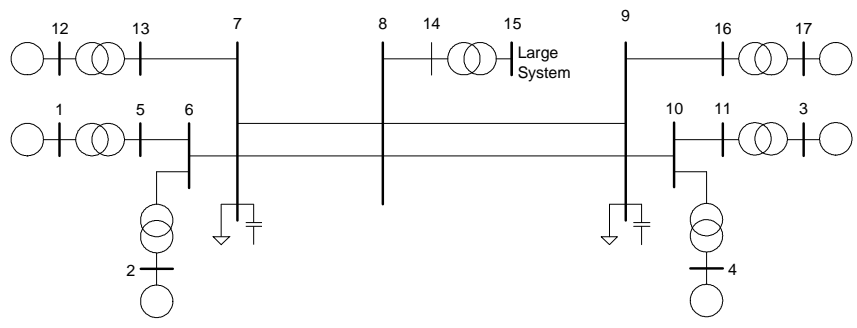

Fig. 3. Test system with three areas.

the lead-lag blocks. The washout time constant is specified previously to the PSS designs and is not adjusted. The weighting factors $w_{j i}$ are used to provide an indication of the best locations to install PSS. Because in this research PSS location is not a concern $w_{j i}=1$ was assumed.

This optimization problem was solved with an evolutionary particle swarm optimization (EPSO) algorithm, inspired in both Evolutionary and in Particle Swarm Optimization, as described in [9].

\section{Results of A CASE Study}

The test system shown in Fig. 3 was considered in this research. The system represents two areas connected to a large system. Each area contains a synchronous equivalent generator (1 to 4), DFIG equivalent wind generation units (12 and 17), a load and a capacitor. The generator connected to bus 15 corresponds to a large system modeled as an infinite bus. Generators 1, 2 and 12 form area 1; generators 3, 4 and 17 form area 2. The large system is considered the third area. Note that generators do not represent a single machine but a group of strongly coupled generators. The system data is presented in appendix.

Synchronous generators are represented using a six-order model, with magnetic saturation neglected, where voltage regulators are included and modeled with an IEEE type 1 model [10]. The DFIG is represented by the model described in section II. Loads are modeled as constant impedance.

Four cases are described next to illustrate the convenience of installing properly tuned PSS in DFIG. The relevant eigenvalues for each case are shown in the pole map of Fig. 4. The electromechanical modes of oscillation are also shown in Table I. The values of real and imaginary component, damping and frequency are included. The last column shows the number of the generator with highest participation in the mode.

\section{A. Without wind power}

Initially, without wind power connected to the system, the system exhibits four modes of oscillation: two inter-area modes with damping around $9 \%$ and two low damped modes with damping of $4.57 \%$ and $3.41 \%$. Mode 3 is characterized by oscillations of all generators against the large system. Mode 4 is described by oscillations of generators located in area 1 against generators located in area 2. The installation of PSS is therefore very much needed.

\section{B. Tuning PSS to install in synchronous generators}

Classical PSS sensitive to speed deviation, to be installed in

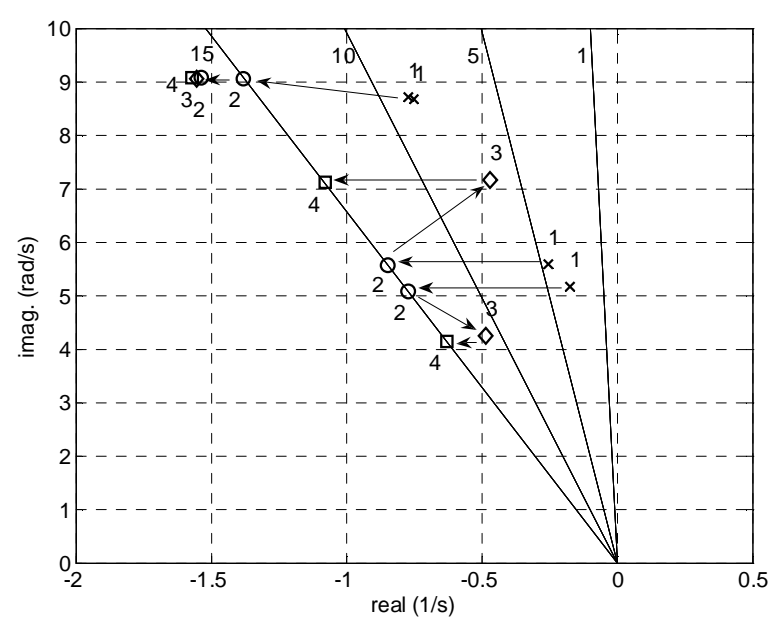

Fig. 4. Pole map. Case number corresponding to each eigenvalue is indicated near the symbol. Diagonal lines indicate damping levels and arrows describe the movement of the modes of oscillation.

TABLE I

ELECTROMECHANICAL MODES OF OSCILLATION

\begin{tabular}{ccccccc}
\hline Case & No. & Real & Imag. & Damp. $(\%)$ & $f(\mathrm{~Hz})$ & Gen. \\
\hline \multirow{4}{*}{1} & 1 & -0.7704 & 8.7059 & 8.81 & 1.39 & 2 \\
& 2 & -0.7518 & 8.6602 & 8.65 & 1.38 & 4 \\
& 3 & -0.2556 & 5.5910 & 4.57 & 0.89 & 1 \\
& 4 & -0.1761 & 5.1680 & 3.41 & 0.82 & 3 \\
\hline \multirow{4}{*}{2} & 1 & -1.3818 & 9.0456 & 15.10 & 1.44 & 1 \\
& 2 & -1.5376 & 9.0671 & 16.72 & 1.44 & 3 \\
& 3 & -0.8485 & 5.5622 & 15.08 & 0.89 & 1 \\
& 4 & -0.7709 & 5.0703 & 15.03 & 0.81 & 3 \\
\hline \multirow{3}{*}{3} & 1 & -1.5524 & 9.0519 & 16.90 & 1.44 & 3 \\
& 2 & -0.4678 & 7.1637 & 6.52 & 1.14 & 2 \\
& 3 & -0.4844 & 4.2538 & 11.31 & 0.68 & 3 \\
\hline \multirow{3}{*}{4} & 1 & -1.5676 & 9.0642 & 17.04 & 1.44 & 3 \\
& 2 & -1.0797 & 7.1084 & 15.02 & 1.13 & 2 \\
& 3 & -0.6274 & 4.1350 & 15.00 & 0.66 & 3 \\
\hline
\end{tabular}

TABLE II

PARAMETERS FOR PSS TO INSTALL IN SG

\begin{tabular}{ccccc}
\hline Gen. & $K$ & $N$ & $T_{n}$ & $\alpha$ \\
\hline 1 & 1.25 & 2 & 1.01 & 30 \\
3 & 1.53 & 2 & 0.95 & 30 \\
\hline
\end{tabular}

synchronous generators, were then tuned using the approach described in section III so that minimum damping increases to $15 \%$. The maximum frequency change allowed was $5 \%$. It was assumed that the installation of PSS is only possible at generators 1 and 3. The PSS parameters are presented in Table II. The damping improvement can be observed in Table I and in the pole map of Fig. 4. Modes are shifted to the 15\% damping line without significant frequency change.

\section{With wind power}

Wind power was then connected to buses 7 and 9 . Generator 1 was switched off to accommodate the new production. The 


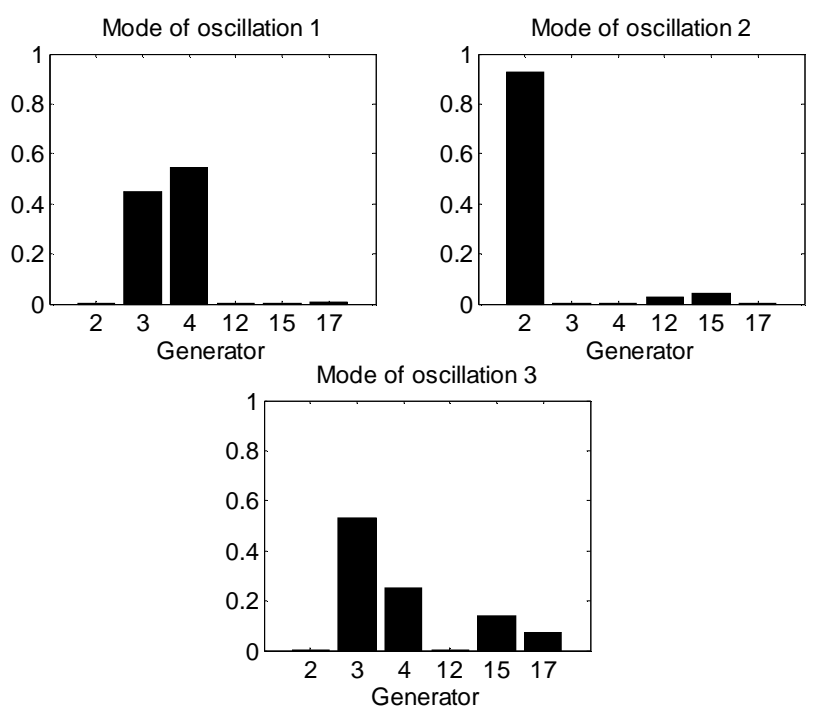

Fig. 5. Participation factors of the electromechanical modes.

remaining generation is absorbed by the large system.

The system exhibits four electromechanical modes of oscillation. Mode 1 is described by oscillations of the generator 3 against generator 4 and is well damped. Damping of mode 2 is $6.52 \%$ which is low. This mode is characterized by oscillations of generator 2 against the system and results from the modification of the inter-area mode of area 1. Note that the additional damping previously provided by the PSS installed in generator 1 is now lost because the generator is offline. Mode 3 shows reasonable damping but bellow the required $15 \%$. The action of PSS installed in generator 3 is present, but the damping decreases due to the higher power flow through the interconnection lines to the large system.

The new eigenvalues introduced by the DFIG are associated to the induction generator and to the converter controllers, and are very well damped. On the other hand, the contribution of the DFIG to the electromechanical modes is not significant.

The contribution of each generator to a mode can be assessed analyzing the participation factors. From Fig. 5 we can confirm that generators 12 and 17 do not participate in the electromechanical modes. In general, if the converter PI controllers are properly tuned the DFIG should not participate significantly in the electromechanical oscillations.

We can then conclude that the reduction of damping is not directly originated by the characteristics of DFIG technology, but is essentially due to the network conditions that favors the emergence of such low damped modes of oscillation.

The system exhibits for this scenario a low damped mode and therefore some action must be considered to increase damping to the specified levels.

\section{Tuning PSS to install in DFIG}

We have already concluded that the mode needing more damping is associated to generator 2. Therefore, the most effective way to increase the damping of this mode is to install a PSS in this generator. Assuming that it is not possible to install new PSS, alternative solutions must be considered.
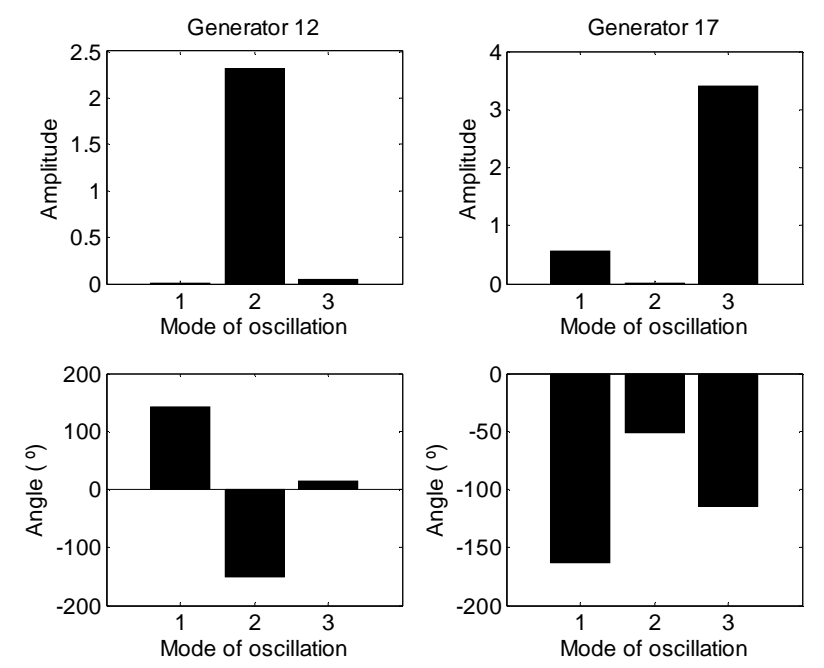

Fig. 6. Residues of generators 12 and 17 for the electromechanical modes of oscillation, transfer function $V_{t} / v_{q r}$.

TABLE III

PARAMETERS FOR PSS TO INSTALL IN DFIG

\begin{tabular}{ccccc}
\hline Gen. & $K$ & $N$ & $T_{n}$ & $\alpha$ \\
\hline 12 & 0.15 & 0 & - & - \\
17 & 0.07 & 2 & 0.10 & 0.57 \\
\hline
\end{tabular}

Because the other synchronous generators, and in particular generator 3, have no participation in mode 2, using the PSS installed in synchronous generators to increase damping is not viable. Retuning the PSS installed in generator 3 is not therefore an interesting solution.

Since the available PSS installed in synchronous generators are unable to provide supplementary damping, the installation of PSS in DFIG is interesting. As described in section II, these devices are able to supply damping provided that observability and controllability exists for the chosen signals. The terminal voltage was selected in this research for the PSS input signal. A rapid measure of the effectiveness of a PSS to damp a mode may be obtained from the residues, witch are the product of observability and controllability factors [11]. Fig. 6 shows the residues of the transfer function from the $v_{q r}$ input to the generator terminal voltage $V_{t}$. The residue is shown for the electromechanical modes and generators 12 and 17.

The residues show that a PSS installed in generator 12 can be used only to damp mode 2 . In the same way, generator 17 will only be effective to damp mode 3 . The angle of the residues of interest is close to $-180^{\circ}$ and therefore the required phase compensation should be small [11].

Classical PSS sensitive to terminal voltage were then simultaneously tuned for both DFIG units. The calculated parameters are shown in Table III. As shown in Table I and Fig. 4 such PSS successfully increase the damping to $15 \%$. Note that, according to the adopted criteria, increasing damping beyond $15 \%$ is not the objective. Design of phase compensation is adequate and frequency change of the mode is small.

The results presented show that, when properly tuned, PSS 


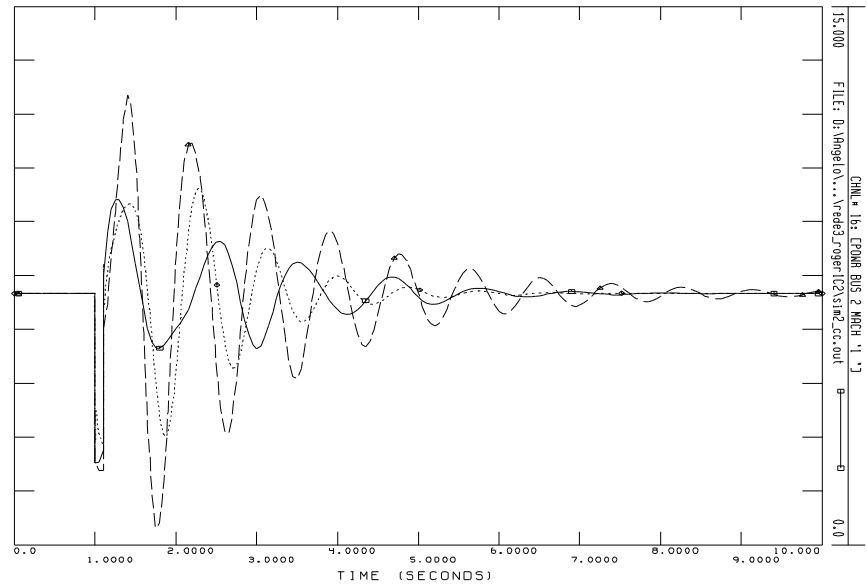

Fig. 7. Electrical power of generator 2 (SG): full line - without wind power (case 2), dashed line - with wind power and no PSS in DFIG units (case 3), and dotted line - with wind power and PSS installed in DFIG units (case 4).

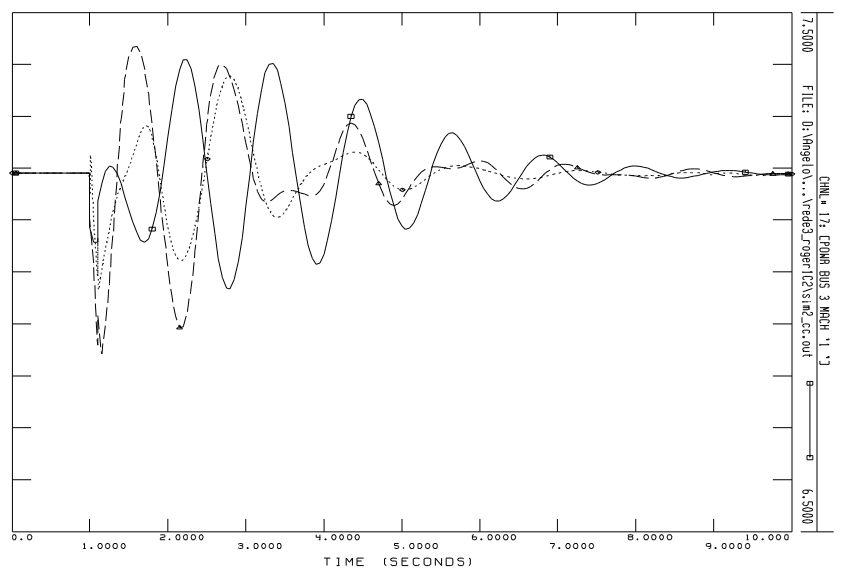

Fig. 8. Electrical power of generator $3(\mathrm{SG})$ : full line - without wind power (case 2), dashed line - with wind power and no PSS in DFIG units (case 3), and dotted line - with wind power and PSS installed in DFIG units (case 4).

installed in DFIG may effectively contribute to increase damping of the electromechanical modes of oscillation. PSS installed in generator 12 is able to increase damping of mode 2 whereas the PSS installed in generator 17 is effective improving damping of mode 3 . It should be pointed out that damping of mode 3 could also be increased by retuning the PSS installed in generator 3 , so that required damping levels are obtained for the scenarios with and without wind power. However, that has no effect on the damping of mode 2 .

\section{E. Time Domain Analysis}

This section presents the nonlinear time domain simulations carried out to validate the PSS tuning results. In addition to the validation of PSS tuning, time domain simulations provide also a clear way to observe the dynamic behavior.

The simulated disturbance was a $100 \mathrm{~ms}$ short circuit in bus 6. Fig. 7 shows the electrical power of synchronous generator 2 for three cases. It is shown that prior to wind power integration the system exhibits a satisfactory behavior due to the action of PSS installed in synchronous generators. After connecting a large amount of wind power the damping of the oscillations deteriorates significantly. The installation of PSS in DFIG units and its proper tuning improves the dynamic behav-

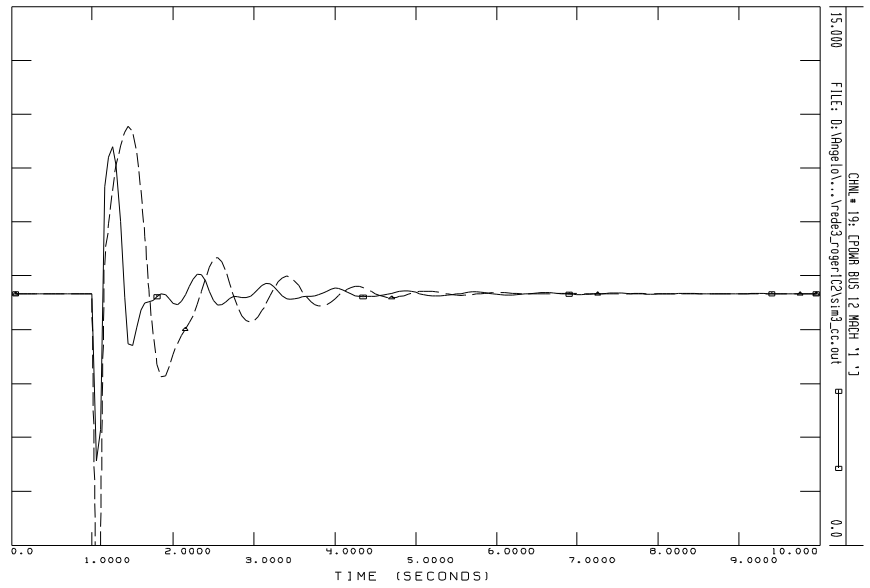

Fig. 9. Electrical power of generator 12 (DFIG): full line - with wind power and no PSS in DFIG units (case 3), and dashed line - with wind power and PSS installed in DFIG units (case 4).

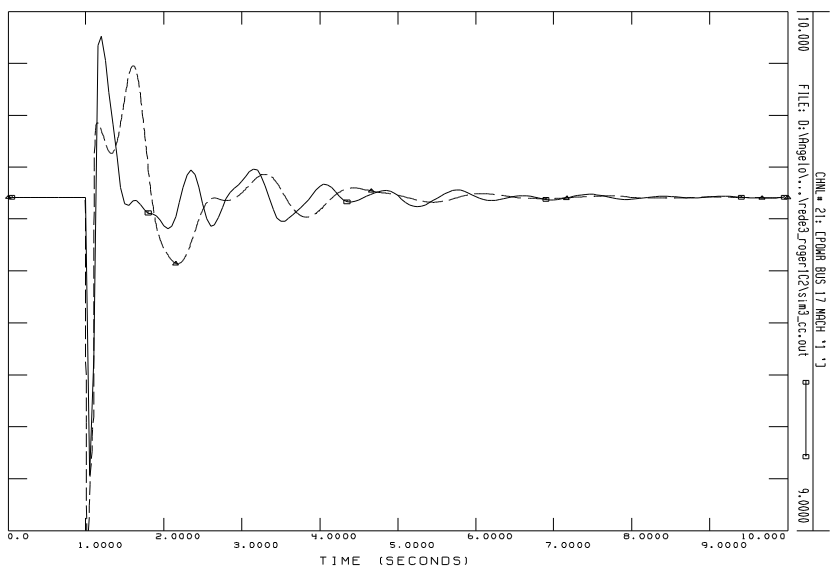

Fig. 10. Electrical power of generator 17 (DFIG): full line - with wind power and no PSS in DFIG units (case 3), and dashed line - with wind power and PSS installed in DFIG units (case 4).

ior of the system and reestablishes the damping levels observed prior to wind power integration. Fig. 8 shows the same results for generator 3 .

Fig. 9 and Fig. 10 show the response of DFIG generators 12 and 17 to the applied disturbance. Although the action of the PSS leads to higher amplitude of the oscillations, especially in the case of generator 12, the dynamic behavior is satisfactory. The increase of the active power oscillations of the wind generators is directly related with the compensation of the electromechanical oscillations provided by DFIG.

We can therefore concluded from these plots that, after installing the PSS in DFIG units, tuned with the parameters identified with the described approach, the system damping is increased considerably.

\section{CONCLUSIONS}

This research shows that properly tuned PSS installed in DFIG wind power generators provide an efficient way to increase the damping of electromechanical oscillations. The effectiveness of the PSS depends on the existence of adequate observability and controllability for the selected signals. The PSS should be properly tuned and the algorithm adopted 
proved to work well. PSS installed in DFIG are therefore a valid option when defining a strategy to increase damping levels of power systems with large wind power integration. As part of that global strategy, such PSS should be coordinately tuned with other devices like PSS installed in synchronous generators or FACTS.

\section{APPENDIX}

\section{A. Network parameters}

$$
\begin{aligned}
& \text { Sbase }=100 \text { MVA } \\
& \mathrm{x}_{8-9}=0.0275 \text { p.u., } \mathrm{x}_{7-8}=0.018 \text { p.u., } \mathrm{x}_{5-6}=0.025 \text { p.u., } \mathrm{x}_{6-7}= \\
& \text { 0.01 p.u., } \mathrm{x}_{7-13}=0.0143 \text { p.u., } \mathrm{x}_{9-10}=0.01 \text { p.u., } \mathrm{x}_{10-11}=0.025 \\
& \text { p.u., } \mathrm{x}_{9-16}=0.0143 \text { p.u., } \mathrm{x}_{8-15}=0.001 \text { p.u.. } \\
& \mathrm{P}_{\mathrm{G} 1}=\mathrm{P}_{\mathrm{G} 2}=\mathrm{P}_{\mathrm{G} 3}=\mathrm{P}_{\mathrm{G} 4}=700 \mathrm{MW} . \\
& \mathrm{V}_{\mathrm{s} 15}=1.03 \text { p.u., } \mathrm{V}_{\mathrm{s} 1}=\mathrm{V}_{\mathrm{s} 3}=1.03 \text { p.u., } \mathrm{V}_{\mathrm{s} 2}=\mathrm{V}_{\mathrm{s} 4}=1.01 \text { p.u. } \\
& \mathrm{P}_{\mathrm{G} 12}=700.3 \mathrm{MW}, \mathrm{P}_{\mathrm{G} 17}=964.2 \mathrm{MW}, \mathrm{Q}_{\mathrm{G} 12}=\mathrm{Q}_{\mathrm{G} 17}=0 \text { Mvar. } \\
& \mathrm{P}_{\mathrm{L} 7}=767 \mathrm{MW}, \mathrm{P}_{\mathrm{L} 9}=1567 \mathrm{MW}, \mathrm{Q}_{\mathrm{L} 7}=\mathrm{Q}_{\mathrm{L} 9}=100 \text { Mvar. } \\
& \mathrm{Q}_{\mathrm{C} 7}=200 \text { Mvar, } \mathrm{Q}_{\mathrm{C} 9}=350 \mathrm{Mvar} .
\end{aligned}
$$

\section{B. Parameters of synchronous generators}

$\mathrm{S}_{\mathrm{n}}(\mathrm{MVA})=900, T^{\prime}{ }_{d o}(\mathrm{~s})=5, T^{\prime \prime}{ }_{d o}(\mathrm{~s})=0.05, T_{q o}{ }^{\prime}(\mathrm{s})=1$, $T^{\prime \prime}{ }_{q o}(\mathrm{~s})=0.4, H(\mathrm{~s})=4, X_{d}$ (p.u.) $=1.75, X_{q}$ (p.u.) $=1.65, X^{\prime}{ }_{d}$ (p.u.) $=0.3, X_{q}^{\prime}$ (p.u.) $=0.75, X_{d}^{\prime \prime}$ (p.u. $)=0.25, X_{l}$ (p.u.) $=$ 0.175 .

$K_{A}=20.0, T_{A}=0.055, T_{E}=0.36, K_{F}=0.125, T_{F}=1.8$.

\section{Doubly fed induction generator}

$$
\left.\left.P_{\mathrm{n}}(\mathrm{kW})=660, \mathrm{~V}_{\mathrm{n}}(\mathrm{kV})=0.69, \mathrm{R}_{\mathrm{s}} \text { (p.u. }\right)=0.067, \mathrm{X}_{\mathrm{s}} \text { (p.u. }\right)=
$$$$
\left.\left.\left.0.03, \quad \mathrm{R}_{\mathrm{r}} \text { (p.u. }\right)=0.0058, \quad \mathrm{X}_{\mathrm{r}} \text { (p.u. }\right)=0.0506, \quad \mathrm{X}_{\mathrm{m}} \text { (p.u. }\right)=
$$
2.3161, $\omega_{\mathrm{s}}$ (r.p.m) $=1500$, No of poles $=4$.

\section{Gains of the PI controllers}

$K_{p 1}=0.10, K_{i 1}=11.29, K_{p 2}=7.96, K_{i 2}=12.50, K_{p 3}=1.76$, $K_{i 3}=13.49, K_{p 4}=12.50, K_{i 4}=1.76$.

\section{REFERENCES}

[1] E. Hagstrøm, I. Norheim, and K. Uhlen, "Large scale wind power integration in Norway and effect on damping in the Nordic grid," in Proc. Nordic Wind Power Conference, Chalmers University of Technology, March 2004.

[2] M. A. Pai, D. P. S. Gupta, and K. R. Padiyar, Small Signal Analysis of Power Systems. Harrow: Alpha Science International, 2004.

[3] G. Rogers, Power System Oscillations. Boston: Kluwer Academic Publishers, 2000.

[4] F. Hughes, O. Anaya-Lara, N. Jenkins, and G. Strbac, "Control of DFIG-based wind generation for power network support," Power Systems, IEEE Transactions on, vol. 20, no. 4, pp. 1958-1966, 2005.

[5] F. Hughes, O. Anaya-Lara, N. Jenkins, and G. Strbac, "A power system stabilizer for DFIG-based wind generation," Power Systems, IEEE Transactions on, vol. 21, no. 2, pp. 763-772, 2006.

[6] R. de Almeida, J. Peças Lopes, and J. Barreiros, "Improving power system dynamic behavior through doubly fed induction machines controlled by static converter using fuzzy control," Power Systems, IEEE Transactions on, vol. 19, no. 4, pp. 1942-1950, 2004.

[7] A. Mendonça and J. Peças Lopes, "Robust tuning of PSS in power systems with different operating conditions," in Power Tech Conference Proceedings, 2003 IEEE Bologna, vol. 1, 2003.

[8] P. Pourbeik and M. Gibbard, "Simultaneous coordination of power system stabilizers and facts device stabilizers in a multimachine power system for enhancing dynamic performance," Power Systems, IEEE Transactions on, vol. 13, no. 2, pp. 473-479, 1998.

[9] V. Miranda, N. Fonseca, "EPSO - Best-Of-Two-Worlds Meta-Heuristic Applied To Power System Problems", in Proceedings of WCCI'2002 CEC - World Congress on Computational Intelligence - Conference on Evolutionary Computing, May 2002.

[10] P. Kundur, Power System Stability and Control, New York: McGrawHill, 1994

[11] L. Rouco, "Eigenvalue-based Methods for Analysis and Control of Power System Oscillations", IEE Colloquium on "Power Dynamics Stabilization”, University of Warwick, Coventry (England), February 1998. 